\title{
Social Mechanisms of Musical Stylistic Change: A Case Study from Early 20th-Century France
}

\author{
JANE HARRISON[1] \\ Istanbul Technical University, MIAM
}

\begin{abstract}
This study examined notated meter changes in scores by French composers to probe the role of sociological mechanisms in musical stylistic change. The stylistic feature of notated meter changes, which indexed metrical complexity, was conducive to empirical observation and functioned as a salient innovation in France around 1900. The principal sociological variable was membership in the Apaches artistic club, known for its avant-garde identity. A hypothesis that the Apaches would use significantly more meter changes than their peers was supported. Additional explanatory variables were derived from previous historical research on French composers and from theories about stylistic change. A complex relationship between the stylistic feature and social mechanisms emerged, involving multiple, overlapping social structures. The Labovian sociolinguistic approach was especially resonant in this data, as a composer's proximity to certain individuals, groups, and institutions in the social space related to their degree of enthusiasm for metric innovation. In addition, sociolinguistic theories about stylistic variation in human languages were consistent with patterns in this data set. Finally, a descriptive title was also a significant explanatory variable, which implicates the Labovian notion of register and the importance that Meyer gave to aesthetic goals in musical stylistic change.
\end{abstract}

Submitted 2020 September 25; accepted 2020 December 19.

Published 2021 June 28; https://doi.org/10.18061/emr.v15i3-4.7469

KEYWORDS: musical stylistic change, sociolinguistics, $20^{\text {th }}$-century France, Apaches

THIS article presents the first stage in what is hoped to be an extensive corpus study project about how processes of a social nature and stylistic patterns in musical creativity might relate. Any socially connected group of composers could be studied for this work. However, we gravitated towards early 20th-century France because several musicologists have already shown how creative decisions were intimately associated with the socio-political structures and processes shaping French society after the creation of the Third Republic government (Fulcher, 1999; Hart, 2008). Of particular interest was the phenomenon of debussyste composers because they seemed to have possessed both rich social and innovative stylistic characteristics. In published articles, interviews, and memoirs, many French music critics and composers active around the year 1900 claimed that many composers were following Debussy's stylistic lead, often referring to them as debussystes. However, no scholar had previously subjected this claim to serious investigation. Using traditional extensive score analysis and examining other primary source material, Harrison (2011) argued that at least a few dozen French composers participated in an identifiably distinctive compositional trend with specific stylistic components. The period of greatest activity seemed to occur between 1900 and 1920. Furthermore, many individuals seemed to adopt debussyste techniques at the same time that they started or strengthened ties with other composers who were already using them, a pattern reminiscent of the role social structures and profiles often play in the diffusion of new ideas and technologies (Rogers, 2003) and in language change (Labov, 1972).

This project essentially takes Harrison's (2011) exploratory findings as a point of departure for a multi-part, empirical case study about musical structure and social structure. As a first step, we looked at a subset of debussystes known for a time as the Apaches (Les Apaches). The Apaches was a club for many types of artists and had Ravel as one of its chief instigators. The club publicly identified as a socially and aesthetically distinct group from their French peers. After sitting together at performances of Debussy's controversial Pelléas et Mélisande during its 1902 premier season, they began to hold meetings in private 
locations. These lasted until sometime early in World War I, at first involving only French-born artists, but eventually including Ricardo Viñes, Manuel de Falla, and Igor Stravinsky. Their main functions were twofold. They would play through and analyze musical scores of other composers that aroused their enthusiasm, in particular the Russian Five and the most recent works of Debussy. As Pasler reports, by 1905 the Apaches were also regularly previewing their own latest works at their meetings to solicit support and feedback from each other (Pasler, 2007). Given the nature of their community, it seemed reasonable to take the Apaches group as a proxy for the debussystes and to hypothesize that the close social proximity of the Apaches members would coincide with some degree of stylistic proximity relative to a control group of their peers.

\section{THEORETICAL CONSIDERATIONS}

The desired approach to the Apaches' creative activity boils down to a corpus study about stylistic features. As Huron (2001) has indicated, the description of musical features is often a foundational aspect of research on music. Yet, anyone who has tried to pin down the features of a musical style understands how difficult it is to define these terms in a consistent and precise way. Huron (2001) notes that the significance of features is ultimately contextual and not absolute, conditioned on a subjective or cultural point of view. Audrey (2008) makes the same point about musical style but through a different conceptualization, by problematizing the long-standing definition of style in art as the manner of expression of the content of a work. Since the content of a piece of music cannot be designated in a single, absolute way, the way in which that content is expressed or joined with a form will likely be difficult to discern as well. Huron (2001) also addresses the fuzziness of musical content and style, finding that significant, interesting musical features are usually patterns derived from the precisely sounding succession of events, such as the perception of chord progressions or variations of a motive. Both scholars help to explain why merely choosing an empirical method such as corpus study is not enough to guarantee valid and useful conclusions about musical style. The methodology is still predicated on a careful decision about what one will look for, based on how those features are meaningful to an identified subset of people. Thus, as Huron says, along with any findings about stylistic features, a framework for interpreting them must also be provided.

In this case, we must begin with a discussion of what is meant by "musical style". Since we are interested in the Apaches as innovators, some potentially applicable principles of stylistic change should also be laid out. Finally, the current research about early $20^{\text {th }}$-century French culture will be added into the discussion, with the end goal being a precise musical feature that can index the possible rebellious creativity of the Apaches. These complex notions cannot be treated in a few sentences. However, their consideration is necessary to the analytical work being carried out, both at this initial stage and as a framework for future studies within the same project.

\section{Definitions of "Musical Style" and "Musical Feature"}

This study is fundamentally about musical style and so that term needs a clear definition. As is well known, the word "style" derives from the Latin "stilus", which in the ancient world referred to both a writing instrument and a mode of expression. This double usage suggests that the writing of a person or group of people contains persistent characteristics that can be perceived as a trace of identity. That notion was also extended metaphorically several hundred years ago to the more abstract or intangible features of someone's manner of verbal expression (Audrey, 2008). Eventually, "style" came to be applied to musical expression, even to music lacking verbal components. Scholars today still widely use the term musical style, although usually without any consideration of its definition. Leonard Meyer was one of the few scholars who attempted to use it more systematically. The definition that begins his touchstone book on style in music is "a replication of patterning, whether in human behavior or in the artifacts produced by human behavior" (Meyer, 1989, p. 3). With "replication" and "patterning" we get important connections to the concept of characteristic features in the etymology of "style", but Meyer does not go on to explain what counts as a pattern or the frequency of replication that makes a pattern qualify as a stylistic feature. He seems to designate significant features of the repertoires he discusses based on his subjective knowledge of specific compositions.

More recently, Huron (2001) has offered some considerations that help fill in the gap in Meyer's seminal treatment of musical style. After he has acknowledged that style is by nature relative, Huron is free 
to treat stylistic features as necessary, imperfect heuristics that should be grounded in precise, consistent observation of operational definitions. This study largely takes the same approach, considering a pattern to be replicated sufficiently to rise to the designation of a feature when it achieves some pre-determined threshold of quantitative significance. Huron also offers some qualities that significant features will need to possess: saliency, concision, understandability, and distinctiveness. While these qualities might seem culturally neutral, they are not. For example, consider European composers of serialist music in the 1950s and 1960s, who strove to engender challenging experiences for performers and listeners (Grant, 2005, pp. 202-221), and thus whose techniques might not be easily described as concise, understandable, or salient. There are music-making communities that emphasize continuity and tradition; therefore, distinctiveness might not be relevant for them. A scholar might be interested in what unites several cases rather than what distinguishes them. Huron's list might be pragmatic for an analyst who wants to describe the distinctive qualities of music examples like a biologist cataloging specimens, which is not without usefulness. His belief that "significance is always contextual, and there is no telling the historical, social, personal, formal or other domains that establish the context for some event-however apparently minor or inconsequential" (Huron, 2001, para. 23) seems, however, to be a surer beginning point than an unconditional list of metafeatures.

\section{Theories of Stylistic Change}

\section{THEORIES FROM MUSIC SCHOLARS}

Huron (2001) has provided the best practical guide for identifying significant musical features. However, he does not consider the historical trajectories of those features or how to incorporate socio-cultural variables into an empirical corpus study. Of particular interest for us were the innovative changes seen in the shared musical conventions of French composers at the start of the $20^{\text {th }}$-century. In our empirical framework, how do we develop appropriate variables to account for the contributing factors or mechanisms driving the changes we see in the score corpora's patterns? Among music scholars, we must again return to Leonard Meyer as the strongest source for a general theory of how stylistic change occurs in musical culture. His definition of musical style as given above still needs its final clause, which states that the replication of patterning "results from a series of choices made within some set of constraints" $(1989$, p. 3). While that initial definition has a ring of hollow structuralism to it, Meyer later goes on to spell out places for individual subjectivity and agency in theoretical notions about the development and persistence of styles. Particularly compelling is the central importance accorded to goals in acts of musical creativity, which drive people to utilize specific stylistic components. He also distinguishes between "rules", which seem synonymous with the term "constraints" in his definition of musical style but are more abstract, and "strategies", which are the more concretely observable manners in which rules are realized. Regarding the nature of lasting changes to the musical style of an entire community, Meyer finds changes to rules to be rare, with strategy changes or innovations being much more common. In any case, a change introduced by an individual or group into the culture might be picked up, Meyer argues, and replicated by many others when it is perceptually salient, can be generalized, and can be used in tandem with other stylistic elements to realize aesthetic goals. This criteria for significant features is preferable to us to Huron's, because it is not beholden to certain aesthetic values, making it applicable to a wider variety of musical cultures; and for our purposes, Meyer's list also drives towards not just a description of musical features but also a framework to account for their historical development.

\section{THEORIES FROM SOCIAL SCIENTISTS}

Starting with the work of Huron and adding in that of Meyer, the concepts of musical stylistic features and change are attaining a clarity that makes them much easier to use. We are still, however, missing systematized thought about the social aspects of this issue, as both Huron and Meyer tend to focus on score analysis and general perceptual constraints on creativity. Concerning the "why" and "how" of the changes in the features displayed by the musical corpora, we turn to Robson and McCartan's (2016) concept of "generative causation", in which multiple mechanisms are discovered to explain why and how something happened instead of a single cause. They state that the mechanisms will be context-specific and are best proposed by people with intimate knowledge of the proper context (Robson \& McCartan, 2016, pp. 32-36). As they placed more emphasis on cultural specificity and contingency since the 1990s, historical 
musicologists have, in a sense, been reaching towards this notion of generative causation. Music scholars also acknowledge the crucial interweaving of social life and musical sounds whenever they interpret an artist's work against details about the "contexts" of their creation. The amount of specific musical analysis varies in such studies, however, and it is rarely up to the standards of systemization of the social sciences. As a result, while many musicologists have a latent sociological point of view, the link between the musical and the social rarely firm up in their scholarship: one cannot proceed from anecdotal evidence in the form of interpretations of specific written and musical documents to generalized conclusions. Logically, then, one does not find general theories about stylistic change in the musicological literature, and findings of different studies cannot be easily compared and amalgamated into future stages of research about overarching questions. Despite these weaknesses, qualitative musicological work is rich in testable hypotheses, variables, and mechanisms that can enliven quantitative, empirical research.

Sociolinguistics is a field that takes stylistic change as its core issue. Since music and language are overlapping domains of embodied human perception (Patel, 2008), sociolinguistics is one highly attractive field in which to look for theories that might apply to style in musical creativity. Some patterns in language sounds have a high degree of regularity because they operate according to regular rules. However, one constantly finds variations in speech patterns from one speaker of a language to the next. Labov, considered to be the seminal scholar of sociolinguistics, argued that while these variations were irregular, the processes giving rise to those variations were actually regular. In many studies of patterns of variations in language communities, sociolinguists have discovered social factors such as socio-economic class, neighborhood membership, or beliefs about the community that act as explanatory mechanisms for very specific variations in how people speak (Labov, 1972; Gordon, 2013).

Possible intersections with the language change findings that have amassed are intriguing. At the same time, there are reasons to be skeptical that patterns of change in this specific corpus of art music scores will overlap with sociolinguistic theories. Composers in the European tradition have often selfconsciously changed the nature of the musical language and practices with which they have worked. That was certainly the case for many composers in early 20th-century France, such as Debussy. Meanwhile, the changes in language style that Labov and other linguists had observed in their studies seem to have resulted from subconscious processes. A certain group of people has started to produce phonemes in a consistent, distinctive way but seem to be minimally or not at all consciously aware of what they are doing or the social variables that connect to them. While such subconscious changing of conventional musical patterns could also occur, in the case of the Apaches it seems more willful. A larger issue is that it is not so easy to speak of music as being generated by a body of consistent rules. Thus, understanding the stable features and the variation within those features could be difficult to identify in musical sound. For the Apaches we would especially doubt such a possibility since members like Ravel and Delage were taking creative steps to gradually dissolve a common practice to music composition in Europe (Gur, 2011).

When he describes how artistic styles change in Art Worlds, Howard Becker (1982) emphasizes the purposefully collaborative nature typical of musicians as opposed to more solitary artists like poets. He seems to prefer the term "characteristic conventions" to "style", and he states that "groups of artists frequently collaborate in the development of innovations so that schools and artistic sects develop characteristic conventions as well" (Becker, 1982, p. 59). As Becker wrote Art Worlds primarily with examples of mid-20th century American and European artists in mind, the more willful, conscious nature of stylistic change in Becker's account might be more appropriate than the emphasis on subconscious innovative acts and patterns in sociolinguistics. In the art worlds and sociolinguistic accounts of change, there is an emphasis on groups of people who feel connected to each other and function as communities. Fortunately, historical research on early 20th-century French art music has yielded many possible specific institutional and other types of cohesive social structures that might relate to developments and variations in the musical features of that repertoire.

\section{INTEGRATION OF MULTIPLE VIEWPOINTS}

From the reviewed authors, we gained several principles to follow as we developed this study's design. A stylistic feature has been defined as a replication of patterning that is described and delimited according to statistical regularity so that it can be submitted to empirical analysis. It can be drawn from the note-to-note sounding surface or a more abstract level of the musical experience. Statistical significance of a feature is only meaningful if it ties to other significances, such as perceptual saliency, with the potential to be generalized and realized in many different concrete strategies and useful for reaching specific aesthetic 
goals or fulfilling specific functions. Huron (2001) has also given us several qualities of musical features to keep in mind. From Labov (1972) and Becker (1982), we have some theories about how stylistic variation from one piece and one composer to the next could be explained by large-scale processes of stylistic change that are rooted in social structures and processes. Following the perspective of musicologists, we believe those properties and processes will be contextualized in a culture. Furthermore, the extant, detailed studies about early 20th-century France are an essential guide for choosing variables, developing questions and hypotheses, and constructing the score corpora. This theoretical apparatus will come together in the story we will attempt to tell about just one feature that appears simple but had powerful cultural and aesthetic ramifications: notated meter changes.

\section{Metric Complexity as a Stylistic Feature}

Harrison (2011) identified several potential innovative debussyste stylistic elements, some easier to submit to empirical observation than others. For the study presented here, we focused only on the general property of metric complexity, by which we mean a situation that is challenging for human meter perception. Experimental and computer modeling research currently suggests that meter is based on mental representations that predict the placement in time of significant events in the musical flow. In real time, people deploy a regularly recurring schema of main beats as a framework to make and evaluate predictive perceptions of what they will hear next (Van der Weij, Pearce, and Honing, 2017). Depending on the characteristics of the music they are heavily exposed to, an adult will become enculturated and therefore perceptually efficient in using only a subset of meter schemas (Hannon, Soley, and Ullal, 2012). The nature of these schemas even differs by culture: while in many cultures of Europe, they consist of regularly spaced beats, in cultures of Turkey and West Africa, the schemas are better described as primary rhythmic patterns (London, Polak, and Jacoby, 2016).

Since we are dealing with composers whose perceptual skills were formed by exposure to 19thcentury art music in Europe, we can deduce that their learned meter schemas would have involved regular beats that were hierarchically nested into groups of two or three, such as in $4 / 4$ or $6 / 8$. Many types of situations could thwart such an anticipatory perceptual framework; as a simple operational definition of metric complexity for this culture, we chose the frequency of changes in the notated meter. More frequent changes were equated with more metric complexity. Our feature of notated meter changes is concise and understandable. However, it ignores the fact that a change or violation of perceived meter can be induced in the musical surface patterns without a notated signature change. Still, it is a good place to start, since it can be easily and consistently observed from one case to the next. Furthermore, copious meter signatures in a musical score have the potential to make a strong visual impression, making it possible for them to serve as meaningful signs for the visual as well as auditory sense like in the various manifestations of "eye music" in the history of European musical notation (Dart, 2011).

Research on music perception has shown how notated meter changes (as well as unnotated ones) tend to be perceptually salient events. Since the meter is so important for the brain and body's ability to predict future rhythms, changing it can create strong feelings of surprise and confusion (Van der Weij, Pearce, and Honing, 2017). In addition to their perceptual meaning, in the culture of early 20th-century France this simple device was markedly non-normative. It is common knowledge among music scholars that French art music composers tended to use a single meter for one movement of a piece throughout the 19th century (and we will see this intuition born out in the data for this study). As a result, changing the meter many times in a single movement functioned as a bold creative choice in the early $20^{\text {th }}$-century, especially before Stravinsky's approach to meter in the mid-1910s became so influential. Bhogal (2013) has connected metric complexity in the solo piano pieces of Ravel and Debussy with a period of increased interest in decorative textures and non-normative experiences of time in early-20th-century French intellectual culture. A score with frequently changing meter can also be tied to Bergson's influential notion of durée, the intuitive and dynamic inner flow of an individual's thoughts and perceptions (Harrison, 2015), and to an interest among certain late- $19^{\text {th }}$-century French artists in the "private organicism" of interior states (Silverman, 1989). Musical time is a remarkable way for humans to represent and instantiate relationships among different aspects of their lives. Strict adherence to the metronome and fixed meter can be understood to represent the sticking to schedules, deadlines, and regulated factory workflows of modern life (van Leeuwen, 1999). If that is the case, when a composer like Florent Schmitt did something as seemingly simple as frequently changing the meter signature in the piano piece Ombres, selected pianists and listeners might have read it as a call for an alternative lifestyle in modern, urban Paris. 
In summary, notated meter changes index rebellious, creative behavior nicely in early 20thcentury France. Of Huron's four qualities of musical features, innovation and individual idiosyncrasy had entered into the range of aesthetic values in French music by this point in time, making distinctiveness a culturally relevant value. We predict that notated meter changes will be found to be distinctive or unusual in our corpora. In addition to its cultural saliency, this stylistic feature is also perceptually salient. Finally, our chosen operational definition of metric complexity is concise, making it easy to observe consistently from one case to the next.

\section{METHODOLOGY}

\section{Hypotheses and Variables}

Since the Apaches considered themselves to be united by a shared desire to rebel, as indicated by the rebellious connotation of their chosen name, we would expect them to be distinctively innovative compared to their peers. Thus, the main hypothesis was that the Apaches would use significantly more meter changes than the control group. After noting that the Apaches were all nearly the same age, a competing hypothesis stating that increased use of meter changes could be better understood to relate to a generation rather than Apaches membership was also tested. Finally, effects of chronological and sociological variables were examined in exploratory multivariate models. All tests were run in $R$ version 3.5.1., and $p \leq .05$ was accepted as statistical significance.

\section{Assembly of Corpora}

To test our hypothesis about the stylistic choices of the Apaches composers, we assembled two corpora of solo piano works. This genre was pinpointed because during this era in France 1) many composers were still writing solo piano pieces, and 2) composers often experimented in this genre (we can think of Ravel's Miroirs or Debussy's piano preludes) more than in conventionalized and/or economically risky genres such as instrumental chamber music or opera. One corpus consisted of all available pieces composed between 1895 and 1925 by the Apaches members Paul Ladmirault, Maurice Ravel, Désiré-Emile Inghelbrecht, Florent Schmitt, and Déodat de Séverac $(n=61)$. We excluded non-French-born Apaches members; André Caplet and Maurice Delage were not represented in the Apaches corpus because they composed 1 and 0 pieces, respectively, in the genre. The control corpus was created by randomly sampling scores available as PDF files on imslp.org from other French composers that had been composed in the same time period $(n=$ 108). A few pieces by Satie, Koechlin, and Enesco that lacked meter signatures entirely were excised; although they are no doubt excellent examples of metric complexity, they lacked the very feature that we used to operationalize that musical quality.

For both corpora, multi-movement pieces were divided. In most instances, each movement was treated as an individual case since European art composers have for several centuries cultivated diversity among the movements in multi-movement pieces. It was important to allow for this typical variation but also not allow pieces with many movements to make an outsized impact on the sample. Movements from a single opus were counted as separate cases for up to five movements unless they were all very short; if the number of movements went beyond five, they were combined through a randomized process so that they were reduced to five cases. This process enlarged the size of the Apaches $(n=138)$ and control $(n=230)$ corpora.

\section{Calculation and Analysis of Meter Change Rates (MCR)}

The number of meter changes was manually counted and recorded for each case and then divided by the total number of measures. Simply counting manually allowed us to retain all of the highly complex scores in our two samples, which would have been extremely difficult to accurately encode and count with computer applications. We refer to this proportion as "Meter Change Rate" (MCR). We used this measurement rather than, for example, the number of incidences per piece, because it functions as a standardized score that permits the comparison of compositions with varying lengths. We multiplied all of the MCR figures, which in their original form were proportions that ranged between 0 and 1 , by 100 in 
order to be able to deal with larger numbers. Thus, each MCR value can be understood as the number of meter changes per 100 measures.

\section{RESULTS}

\section{General Considerations}

The data analysis presented here tries to meld together scientific empirical and humanistic philosophies; the first one emphasizes a pre-planned, tightly controlled framing of the inquiry while the latter one encourages a more open interaction with the data in which the steps emerge one out of the other. Since both approaches have their strengths, statistical tools have been utilized in the manners of both. Two rigorous hypothesis tests were undertaken, followed by other tests that unfold as a kind of journey taken into the data as one test brought out a certain question that a subsequent test tried to answer. This journey might be criticized as post hoc testing, but we believe that it is true to the traditional musicological emphasis on multiple viewpoints and storytelling as necessary to understanding complex musical situations. Finally, this text includes some explanations of statistical techniques for the music scholars out there who might be interested in the findings but are not very familiar with statistics; for the readers who are already knowledgeable about these tests, we ask for their patience.

\section{Basic Tests of the Hypothesis of Difference Between Two Groups}

We tested the hypothesis that the Apaches would use significantly more notated meter changes than the control group. Although we were convinced that they are representative of their populations, the distribution plot in Figure 1 displays how both the Apaches and control samples are right-skewed, due to a large number of cases with zero meter changes.

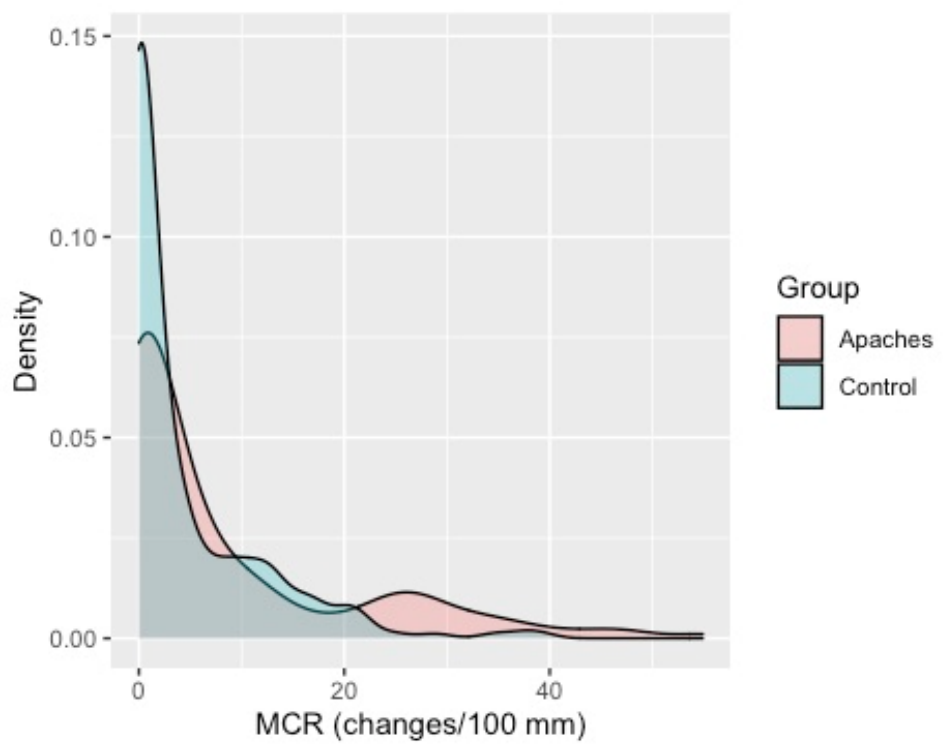

Figure 1. Distribution plot of MCR by group. Kernel density plot made with the density command using its default settings in the stats package of R. It can be understood as a smoothed-out histogram of the frequency distribution. The MCR values in both groups are strongly right-skewed.

There are debates about the extent to which the t-test is compromised by non-normal distributions. Therefore, we instead applied a Mann-Whitney-Wilcoxon test, which does not involve any central tendency measures. A one-sided test was applied since the hypothesis was directional. The rate of meter changes per 100 measures in the Apaches group $(M d n=3.15, M=8.55, S D=12.33)$ was shown to be higher than that 
of the control group $(M d n=0.80, M=4.47, S D=7.12), W=18,338, p=.004, r=.14$. This significant result's small effect size can be seen from the fact that the $r$ statistic is below .30 .

The potential outsized impact of Florent Schmitt, who early in his career composed an especially large body of solo piano pieces, must be assessed. He contributes 55 out of 138 cases in the Apaches corpus. Schmitt's impact can be simply assessed by removing his pieces and re-running the one-sided Mann-Whitney test between the two groups $(W=11,152, p=<.01, r=.14)$. The hypothesis test that the Apaches composed with more notated meter changes than the control sample was supported without Schmitt's contribution, but again there was a small effect size for this difference.

The results of the Mann-Whitney tests mean that if the MCR values for both groups are ordered from lowest to highest, the Apaches' values consistently fall higher in the rankings than those of the control group. That test, however, is only one operational definition of difference, and it excludes all of the cases with zero meter changes. It also only addresses the MCR data points as positions relative to each other and not as precise values. We wanted to better understand the differences between the two sets of numbers with a more nuanced point of view. A second look at Figure 1 shows that both distributions contain multiple curves, which make it difficult to describe them with a single mathematical function or a single central tendency measure like a median. As a result, we decided to compare the two groups' data within various sections of the MCR range. Therefore, we created three partitions based on the mean and standard deviation $(S D)$ of the nonzero values of both groups: "low" - values at or below the mean $(0.01-11.04)$, "medium"- values between the mean and the mean plus $1 S D$ (11.05-21.69), and "high"-all values above one $S D(21.69+)$. A fourth partition was designated for all cases with precisely zero MCR. We determined these boundaries before running Chi-Squared tests and did not alter them post-hoc. Each case was coded as falling into one of these four partitions (zero changes; low, medium, or a high number of changes). Chi-Squared tests showed associations between group (Apaches vs. control) and the number of scores falling into the different partitions, $X^{2}(3, N=386)=18.57, p<.01$. Inspection of standardized residuals for values above 2 or below -2, following the methodology outlined by Agresti (2002), showed a very strong association in the "high" range, and indeed the Apaches' scores were six times more likely than those of the control group to contain more than 21 meter changes per every 100 measures. No other residuals were significantly large.

The MCR data of both groups is notable for its high number of zeroes (168 out of 368 movements). Rather than a flaw in the data set that needs to be altered or discarded, we understood those zeroes to be accurate and important information about the culture under study. Having so many of them is consistent with the common belief that around the year 1900, French composers usually took the meter signature to be something designated one time and then left in place. Given this demonstrable general lack of metric flexibility within this culture, the use of even a few meter changes in one composition becomes a remarkable act. Seeking a test that would meaningfully take account of the high number of zeroes, the hypothesis about Apaches membership and the frequency of notated meter changes was tested once more using a zero-inflated negative binomial (ZINB) model. Since the negative binomial distribution used for the count portion of the ZINB model cannot accommodate the decimals in our MCR values, they were all rounded to the nearest integer. All values greater than 0 but less than .5 were rounded up to 1 , so that 0 would only mean the utter absence of any changes.

A ZINB model consists of two parts, a binomial zero/non-zero and a negative binomial count portion. Each zero in the data set can fall into two latent classes, one for cases that would always score zero (binomial portion) and another for those that would score zero only under certain conditions (count portion). This latent division in the pool of zeroes should be justified by a real-world reason (Faraway, 2016, p. 94). In the case of early 20th-century French art music, in some compositional situations, such as for a certain dance type, it would not have been appropriate to change the meter. In addition, a particular composer might have consistently avoided meter changes throughout his or her career under the influence of the cultural norm of metric stability.

The $p$-values of the ZINB model in Table 1 show that the Apaches versus control variable was a significant single predictor for the count component - with the Apaches MCR values being on average $69 \%$ higher than those of the control — but not for the binomial component estimating the probability that a movement will have zero notated meter changes. If we consider that $41 \%$ of the Apaches scores and $49 \%$ of the control scores had no notated meter changes, this result makes sense. We will return to the question about which other variables might explain the zero-inflated portion of the data in a further set of ZINB models. 
Table 1. ZINB Model of Rounded MCR Against Apaches/Control Variable Alone.

\begin{tabular}{llll}
\hline & Exp. Coefficient & SE & $P$ \\
\hline Y-intercept & Count & & \\
Group (Apaches) & 7.85 & .10 & $<.01$ \\
Log (theta) & $<.01$ & .15 & $<.01$ \\
& & .16 & .99 \\
Y-intercept & Zero-Inflated & & \\
Group (Apaches) & .74 & .17 & .08 \\
& 1.36 & .25 & .22 \\
Theta & 1.00 & & \\
Log-likelihood & -917.9 on $5 d f$ & & \\
AIC & 1845.82 & & \\
\hline$N$ & 368 & & \\
\hline
\end{tabular}

\section{Hypothesis Test with a Modified Control Group}

We have now seen many statistical tests in which the five Apaches composers handled notated meter changes differently from the control group. It is important to precisely interpret those findings based on our sampling method. Compiled according to a randomized process that involved pieces by a large and diverse pool of composers, the control group is a robust construct for observing how composers were generally handling meter signatures in early 20th-century France. At the same time, limiting the control composers to a maximum of five whole compositions but including so many pieces from the Apaches gave them an unfair advantage of sorts in the statistical tests. If the control group was given a similar opportunity to show its highest MCR scores, perhaps the Apaches would not appear so daring. We looked at all of the MCR values in the data set and chose the five control composers whose scores posted the highest single numbers; those composers were Maurice Emmanuel, Gabriel Dupont, George Enescu, Albert Roussel, and Jean Roger-Ducasse. Then we culled all of their solo piano pieces written between 1890 and 1925 that were available on IMSLP.org. Multi-movement compositions were treated as outlined previously. There were $n$ $=135$ Apaches and $n=83$ Others movements. Although the mean of the Apaches group $(M=8.5, S D=$ $12.11, M d n=2.78)$ was higher, the median was actually lower than that of their five challengers $(M=7.10$, $S D=8.47, M d n=4.44)$. We tested the hypothesis that the Apaches would compose significantly more notated meter changes than the Others with a one-sided Mann-Whitney-Wilcoxon, which showed that the difference in medians was not significant, $W=5,041, p=.66$. A Chi-Squared test on the association between the four-fold partition of the MCR range specified above and the Apaches/Others was significant, $X^{2}(3, N=218)=8.52, p=.04$. When we examined the standardized residuals and then the risk ratios, we saw that the test result was driven especially by the fact that the Others were 2.26 times more likely than the Apaches to write a composition whose MCR was in the "medium" range (11.05-21.69). To examine the possibility that the Others might significantly outperform the Apaches in general, we ran the alternate one-sided Mann-Whitney-Wilcoxon test. The result was not significant, $W=5,041, p=.34$. At this point it can be said that, although the initial hypothesis of significant difference between the Apaches and a very generalized control group of their peers was supported, a modified version of that hypothesis in which the control group was more specific and competitive was not supported.

\section{Exploratory Analysis of Other Covariates}

Since the Others subset of the control is quite well-matched to the Apaches, we conducted exploratory analysis to pinpoint additional explanatory variables for the variation in the MCR data. We also still needed to refine our initial ZINB model. Comparing the Apaches' and Others' Tukey's Five Numbers, which indicate the center and the extremes in a data set, add greater nuance to how the creative choices of the 10 composers relate to each other. They also reveal some important disparities among the Apaches. As shown 
in Table 2, the maximums of Inghelbrecht, Ravel, and Schmitt stand out as extreme, but Ladmirault and Séverac fit better with the 5 Others on this point. Inghelbrecht peels away from Ravel and Schmitt if we look at their minimums and lower quartiles, showing that he more consistently composed with notated meter changes across his works than they did. By those measures, Inghelbrecht actually has more in common with Dupont and Ducasse. Despite being one of the first composers to join the Apaches, Ladmirault's consistently less adventurous approach does not fit well with those of the other members. Interestingly, he was also socially distinctive from them on a few major points. Originally from the Bretagne region of France, in 1920 he returned to his birthplace of Nantes while the other French Apaches remained in Paris. By 1910 Ladmirault had also become a founding member of "Les Huit", a group of nationalistic Breton composers.

Table 2. Tukey 5-Numbers for Apaches and Others (5 other highest MCR scorers in data set).

\begin{tabular}{llllllll}
\hline Composer & DOB & Group & Min. & $\begin{array}{l}\text { Lower } \\
\text { Quartile }\end{array}$ & Median & $\begin{array}{l}\text { Upper } \\
\text { Quartile }\end{array}$ & Max. \\
\hline Ducasse & 1873 & Others & 0.00 & 2.86 & 5.71 & 8.24 & 24.37 \\
Dupont & 1878 & Others & 1.33 & 6.98 & 12.99 & 18.60 & 28.89 \\
Emmanuel & 1862 & Others & 0.00 & 0.00 & 6.21 & 14.74 & 18.60 \\
Enesco & 1881 & Others & 0.00 & 0.00 & 0.00 & 4.35 & 21.00 \\
Inghelbrecht & 1880 & Apaches & 0.00 & 4.50 & 12.50 & 27.72 & 54.95 \\
Ladmirault & 1877 & Apaches & 0.00 & 0.00 & 0.00 & 3.56 & 10.78 \\
Ravel & 1875 & Apaches & 0.00 & 0.00 & 0.32 & 11.05 & 47.87 \\
Roussel & 1869 & Others & 0.00 & 0.00 & 0.00 & 7.99 & 37.35 \\
Schmitt & 1870 & Apaches & 0.00 & 0.00 & 3.15 & 10.00 & 45.80 \\
Séverac & 1872 & Apaches & 0.00 & 0.00 & 1.21 & 5.24 & 27.84 \\
\hline
\end{tabular}

Table 2 also shows that the Apaches were all born between 1870 and 1880, and the five Others composers also fall into or very close to that range except for Maurice Emmanuel. This suggests that generational membership might subsume the Apaches variable and therefore better explain the MCR data. To examine that possibility, we widened the time window out to $1865-1885$ and then coded each controlgroup composer's birth year as falling inside it or not. A one-sided Mann-Whitney-Wilcoxon test showed that the Apaches' MCR values were not significantly greater than those of their generational peers who were not in their club $(W=8,188.5, p=.11)$. The Apaches' MCR data is therefore not very different from that of other composers of their generation, meaning that to a certain extent, they were taking part in a trend that influenced a certain age group. This was indeed an era in French musical culture when notions of youth and modernity gained symbolic capital, and therefore following trends became an issue. Scholars have already documented examples of groups of composers desiring to distinguish themselves in public discourse as young people entering their field with relatively new points of view, such as Les Six or the tellingly named La jeune France. Jane Fulcher (2005) has written critical historical accounts of both of those groups, for example.

A Chi-Squared test of the association between the MCR range partitions used earlier and the Apaches versus non-Apaches 1865-1885 generation was significant $\left(X^{2}(3, N=247)=16.48, p<.01\right)$, and when we examine the standardized residuals and risk ratios a complex situation emerges. While the Apaches were nearly five times more likely to reach the "high" MCR level, their generational peers were 2.4 times more likely to reach the "medium" range. When we explore the possibility that the strong showing of the Others control subset can be better explained as a generational effect, we see mixed results. This variable is somewhat successful, although the Apaches show a marked and important difference from their generational peers in their attraction to changing the notated meter with extreme frequency.

The Apaches' extreme behavior regarding meter is also displayed in the innovative meter signatures that one finds in their scores more often than in either the Control group or the Others subset of the control. Some examples are shown in Figure 2, below. The Apaches employed such inventions in select pieces that also contain very high numbers of meter changes. In contrast, when we inspect the control pieces with the highest MCRs, very few of them contain unusual or novel signatures; they instead show high numbers of the traditional signatures like 4/4, 3/4, and 6/8. The one exceptional control-group 
composer who does behave like the Apaches in this respect is René-Emmanuel Baton, born in 1879, just one year before Inghelbrecht.

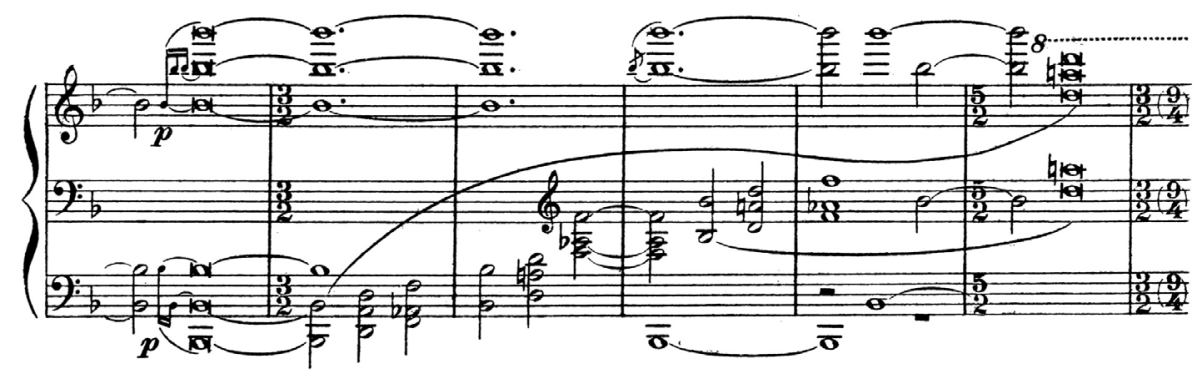

Figure $2 a$. Frequent meter changes and metric ambiguity denoted by an innovative double signature in D.E. Inghelbrecht, Paysages, "La lune sur la plaine," mm. 5-10. First measure is in 5/2.

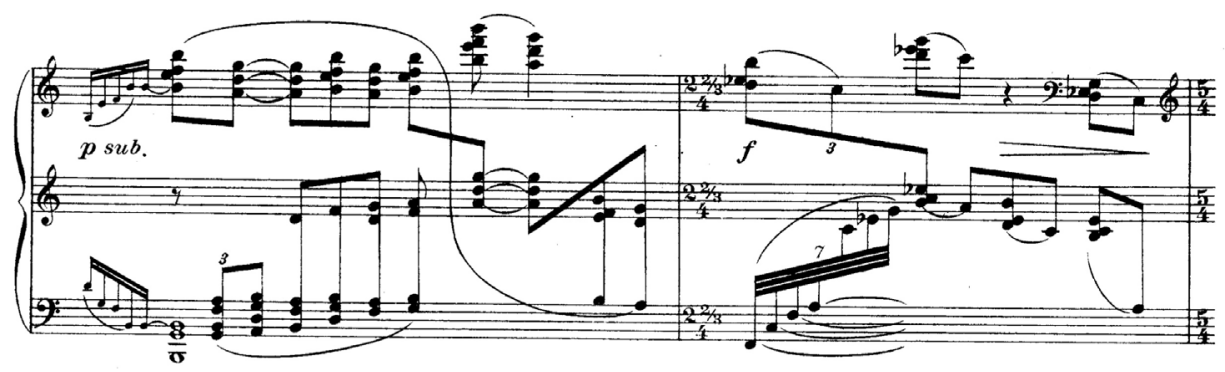

Figure $2 b$. Innovative meter signature containing a fraction in Florent Schmitt, Ombres, "J'entends dans le lointain", mm. 123-124. First measure is in 4/4.

Stylistic change cannot be properly examined without temporal variables. The scatterplot in Figure 3 depicts the MCR values against the composition date of the pieces in the Apaches group, the control group composers that share the Apaches generation (1865-1885 Gen), and the remaining others in the control group (Other Control). Jitter was added to the data for better visualization of the distribution of the points with very low values.

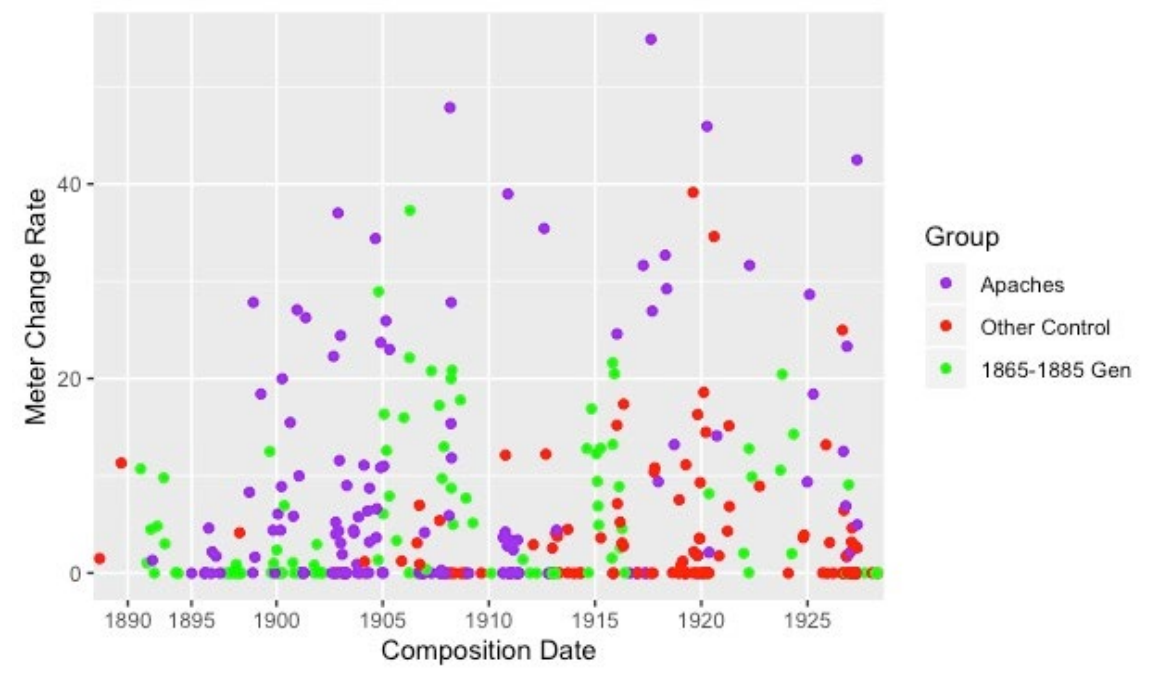

Figure 3. MCR data plotted by composition date according to three different social groups; the three groups form their own distinct waves of increasing scores. 
Based on the constant presence of MCR scores around 2 along the x-axis, which indicated two meter changes per 100 measures, it seems safe to say that between 1889 and 1925 , it was culturally permissible for a French composer to change the meter in a composition a handful of times, especially between closely related signatures. See, for example, Bachelet's insertion of a few $9 / 8$ bars in his Barcarolle Nocturne (1889) that is otherwise in 6/8. The Apaches MCR values arch up over this low-level activity starting around 1895 and reach relatively high values by the first years of the next century. The 1865-1885 generation shows a similar trend that lags by about five years, while the rest of the control group finally shows a modest peak in values around 1920 .

Now is a good moment to refine our ZINB model-especially the portion that predicts the zeroes - by adding in chronological and other additional variables. Table 3 shows two models that we constructed and compared. First, we will describe the three new variables present. Composition Date and Birth Year are self-explanatory. Schola (Schola Cantorum) and Cons (Paris Conservatoire) refer to the two music schools in Paris at which nearly all the composers in our data set spent time as students and/or teachers. In the musicological literature, the environment at the Schola Cantorum is often cast as more conservative and constraining than that of the Paris Conservatoire (Woldu, 2001). Thus, we wondered if that supposed tendency would be consistent in the meter change data. Since some composers attended both schools or studied at one school but taught at the other, we decided to treat them as separate binary variables that encompassed both types of affiliation. "TitleDesc" (Descriptive Title), means that instead of being titled after a standard genre (e.g., "Sonata" or "Prelude") or dance type (e.g., "Valse-Ballet" or "Rigaudon"), a movement's title was more specific, like a character's name or a detailed description of a scene.

Since the 1865-1885 generation variable ("Gen") showed itself to be so competitive with the Apaches variable, we wanted to see how they would compare in a multivariate model. Due to their strong correlation with each other, they could not be combined into the same model, so we instead ran two models with identical constructions except for the presence of Apaches in Model A and Gen in Model B. We will avoid extensive interpretation and keep to a few general remarks about these models, since they are not based on data that was gathered specifically for their formulation. The two models show similarities: The Apaches and Gen variables contributed positive coefficients in both parts of the model, although only the Apaches variable in the count portion was statistically significant, and for both variables the only significant interaction term was with TitleDesc. According to AIC scores, while Model A and Model B are both improvements over the single-effect model in Table 1, Model B has a clear advantage over Model A. On the whole, membership in the 1865-1885 generation and in the Apaches circle function equally well as main effects on MCR.

Both the Apaches and Gen variables have a positive effect not just on the magnitude of the notated meter change rate but also on the probability of having zero changes. The two statements are not mutually exclusive, but this situation is not ideal for a ZINB model. Their interaction term with TitleDesc helps to clarify what is happening: in both models' zero-inflated portions it is significant, whereas Apaches and Gen alone are not. The direction of the interaction terms' effect is in both cases negative and close to a factor of .25 , meaning that if a composer of either membership utilizes a descriptive title, they are much less likely to exclude notated meter changes entirely from their composition. TitleDesc also plays a significant role in the count portion, as a main effect in Model A and in the interaction term in Model B.

Their multiple significant $\mathrm{p}$-values for Schola and Conservatoire affiliations indicate that they are good candidates for future studies on style and social life in early $20^{\text {th }}$-century France. Their comparison shows that they both consistently contribute negative effects of similar magnitude in each of the four model parts shown in Table 3. These results cast doubt on the contention that the Schola Cantorum can be considered definitively conservative compared to the Paris Conservatoire, making it all the more useful to probe the two institutions' effects on stylistic variables in further testing. Finally, the effects of both the chronological variables (birth year and composition date) were very small throughout both models, with coefficients that hovered consistently close to 1 . The performance of these variables emphasizes that notated meter changes were not simply becoming more frequent with the passing of time, but that the variation in MCR depended on other factors. 
Table 3. Multivariate ZINB Models of Rounded MCR Values

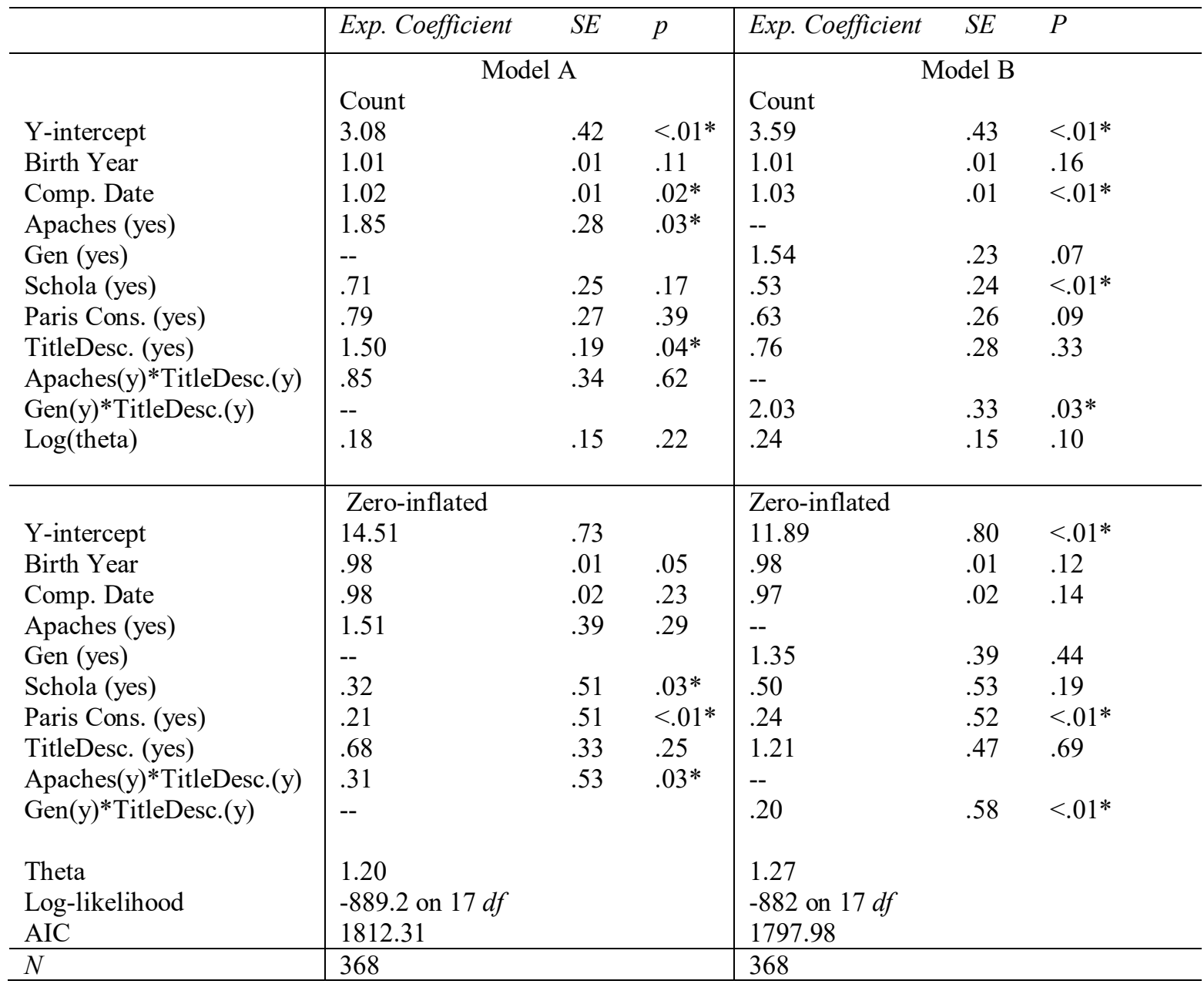

\section{DISCUSSION OF RESULTS}

This methodological design attempted to combine the perspectives of multiple disciplines to conduct a score corpus investigation of musical stylistic variation. In addition, a major aim of this work was to keep sight of the "real world" situation to which the data referred, especially by keeping in mind the heuristic of mechanism and seeking out conceptualizations of variables tailored to these composers' lives. The large body of diffusion of innovations research shows that social networks are an important mechanism through which innovations spread or are prevented from spreading from one person to another (Rogers, 2003, p. 19). In the case of composers in turn-of-the-century France, institutional affiliation, Apaches membership, and generational cohort are all examples of network mechanisms that related to metric innovation. Statistical analysis has suggested that some network dimensions were more effective conduits for spreading the practice of changing meter than others. For example, a composer's generation had a stronger association with the stylistic variable than the school they attended.

The social fact of Apaches membership did exert a significant effect on the rate of meter change, as predicted. However, the subsequent chain of inquiry made clear that as variables affecting metric complexity, multiple divisions of the social space were meaningful. Particularly revealing was the similarity between the Apaches and the five other high-performing composers, who were seen to have generational cohort in common. If we look at the music historical sources, we can see that these ten composers had still more social linkages: many of them crossed paths in salons such as that of Madame Saint-Marceaux (Chimènes, 2004), in Fauré's composition class, and as founding members of the Société 
musicale indépendante (Duchesneau, 1997). Thus, the limitations in looking for stylistic differences among disjointed groups have been clearly exposed. The Apaches were subsumed by a much larger, more complex network, which cannot be examined without a more sophisticated analytical technique such as social network analysis.

Despite this study's preliminary nature, the sociolinguistic paradigm was shown to be relevant to stylistic variation in music in several ways. Labov's very conceptualization of stylistic variation in language also relates to what was seen here: the stylistic behavior is not on or off but rather manifests at multiple points along a continuum of more and less extreme presentations. Just as some people shift a vowel sound a little bit while others shift it a lot, some individual composers employed changing meter more often and/or in more extreme forms than others. Furthermore, sociolinguists have consistently found that degree of variation of specific speech sounds relates to highly specific social network variables; Labov, for example, found in his seminal study about Martha's Vineyard that degree of centralization in diphthongs among the residents related to their ethnic affiliation, home location on the island, and even their feelings of loyalty to the community (Labov, 1972, pp. 27-39). In the same way, an early 20th-century French composer's proximity to certain individuals, groups, and institutions in the social space relates to their enthusiasm for metric complexity and innovation. The Apaches, for example, were shown to be an especially extreme group regarding this behavior according to multiple measures, while other composers who were not in the group but associated with some of the members showed a remarkably similar propensity. Composers at greater social distance - those control composers not in the Apaches' club or their generational cohort - tended to also be stylistically distant in terms of metric innovation. A social network analysis map would likely make these network relationships to stylistic variables much more precise and would also allow us to ask whether something like Labov's notion of "speech community" (ibid., pp. 120-121) can be functional in musical creative practices.

In both of the final two multivariate models of MCR values, the descriptive title variable played a surprisingly strong role. Recalling why notated meter changes were chosen as the stylistic variable, frequent meter changes impart a dynamic, fluid sense of time to pieces of music, and in fin-de-siècle France such a sense of time was associated with a counter-cultural way of life in in which fleeting, subtle interior states and their expression in art were highly valued. In a similar way, descriptive titles display a composer's intention to break out of the normative functions, characteristics, and procedures of a genre, and to instead impart a relatively unusual and specific experience. The positive effect on notated meter changes of that variable in four places in Table 3 can be taken as empirical support for the link that historical musicologists like Bhogal (2013) and Harrison (2015) have made between the rule of metric complexity with this precise aesthetic goal. The positive effects on MCR of the interaction terms of the Descriptive Title ("TitleDesc") with both Apaches and 1865-1885 generation ("Gen") membership add to this picture, suggesting that composers in those social networks seemed to be especially in tune with the cultural movement towards subtlety in France around 1900. The social, stylistic, and aesthetic combine in one phenomenon.

Along with indicating what the music is about, descriptive titles direct people towards specific usages and situations of consumption, just as generic titles do. Therefore, the strength of the TitleDesc variable in the models is suggestive that stylistic variation in music could involve something like the concept of register in speech as developed by Labov, in which individuals do not speak a language uniformly but noticeably adjust many aspects of their language production in a consistent way depending on the genre or situational context of what they are saying (Gordon, 2013, p. 103). While single-variable tests on notated meter change frequency pointed in the direction of the importance of social structural relationships and positions, the significance of the situation and intended meaning of the composer's creative work was shown to be at least as important in multivariate analysis.

Becker's theories (1982) about stylistic change were also born out in our data, in that the Apaches indeed seem to be an example of a group of artists collaborating to effect stylistic innovation. It also seems appropriate to designate the Apaches as a "sect" following Becker, given their empirically verified extreme stylistic behavior. The term "sect" must be criticized, though, since it became clear that the Apaches were part of a much larger, fluid network of composers and were not the only metric innovators within it. This concept shows limitations similar to that of compositional school (i.e., "Romantics") as an explanatory variable for stylistic variation. Becker's emphasis on the role of institutions in the development of artistic style was also consistent with the significant relationship between meter change behavior and music school affiliation. 
Looking back over our results, it becomes clear that Meyer's (1989) comments on style are directed to a complex level of any narrative about musical stylistic change. It is a level that is mostly beyond the scope of these results: because we looked at only one strategy for the rule of metric complexity, robust contentions about the rule level cannot be put forth. We can, however, see a structural relationship among strategy, rule, and aesthetic goal in these findings, especially in the discussion about descriptive titles. A major question about the Apaches and their close peers is whether they were participating in a paradigm shift, as Harrison (2011) essentially argued, in which composers were favoring complexity and novelty in multiple aspects of their music at once. We can find examples of ambiguity in the domains of tonal function, timbre (i.e., confusion about sound sources and manner of sound production), voice leading, and meter in a single composition such as Debussy's Nuages; how widespread was that approach? The prevalence of such stylistic constellations must be carefully empirically assessed, and then scholars can be in a position to think at the level of Meyer's theory: were multiple stylistic innovations being combined to serve a unified aesthetic goal? How does metric complexity fit into that broader history? To what degree and in what ways did composers generalize and create strategies for metric and other types of complexity? Meyer has suggested an impressive research program to address key issues about stylistic change in music, indeed!

In future studies, it is hoped that the theoretical bases of Labov, Becker, Meyer, and Huron will be brought together with greater sophistication to glimpse the interaction of social variables of institutional, network, and demographic types with stylistic features, strategies, and rules. Certainly, metric complexity in this data set needs to be examined in its common other forms, including unnotated meter changes, hemiola, and syncopation. Although meter changes might seem like a simple dimension of a musical score, music psychologists and semiologists have elucidated the perceptual and phenomenological salience of a lack of metric stability. Historical musicologists have uncovered the aesthetic meaning that this experience had for composers in early $20^{\text {th }}$-century France, and this study has offered some evidence that there was also a complex sociological dimension to this cultural phenomenon.

\section{ACKNOWLEDGEMENTS}

This article was copyedited by Annaliese Micallef Grimaud and layout edited by Diana Kayser.

\section{NOTES}

[1] Correspondence can be addressed to: Jane Harrison, Istanbul Technical University, harrison@itu.edu.tr.

\section{REFERENCES}

Agresti, A (2002). Categorical data analysis ( $2^{\text {nd }}$ ed.). Hoboken, NJ: Wiley. https://doi.org/10.1002/0471249688

Audrey, E. (2008). Reconsidering high style and low style in medieval chanson. Journal of Music Theory, 52(1), 75-122. https://doi.org/10.1215/00222909-2009-011

Becker, H. (1982). Art worlds. Los Angeles, CA: University of California Press.

Bhogal, G. (2013). Details of consequence: ornament, music, and art in Paris. Oxford, UK: Oxford University Press.

Chimènes, M. (2004). Mécènes et musiciens: du salon au concert à Paris sous la IIIe République. Paris, France: Librairie Arthème Fayard.

Dart, T. (2001). Eye music. In Grove Music Online 2001. https://doi.org/10.1093/gmo/9781561592630.article.09152

Duchesneau, M. (1997). L'Avant-garde musicale et ses sociétés à Paris de 1871 à 1939. Wavre, Belgium: Editions Mardaga.

Faraway, J. (2016). Extending the linear model with $R$ ( $2^{\text {nd }}$ ed.). Boca Raton, FL: CRC Press. 
Fulcher, J. (1999). French cultural politics and music: from the Dreyfus affair to the First World War. Oxford: Oxford University Press.

Fulcher, J. (2005). The composer as intellectual: music and ideology in France, 1914-1940. Oxford: Oxford University Press.

Gordon, M. (2013). Labov: A guide for the perplexed. New York, NY: Bloomsbury.

Grant, M. J. (2005). Serial music, serial aesthetics: compositional theory in post-war Europe. Cambridge: Cambridge University Press.

Gur, G. (2011). The spectre of the end: Musical avant-gardism and the philosophical turn. International Review of the Aesthetics and Sociology of Music, 42(2), 267-284.

Hannon, E., Soley, G., \& Ullal, S. (2012). Familiarity overrides complexity in rhythm perception: A crosscultural comparison of American and Turkish listeners. Journal of Experimental Psychology: Human Perception and Performance, 38(3), 543-548. https://doi.org/10.1037/a0027225

Harrison, J. (2011). Fashionable innovation: Debussysme in early twentieth-century France. Unpublished doctoral dissertation, The Ohio State University, Columbus, $\mathrm{OH}$.

Harrison, J. (2015). The spiritualiste connection: Stylistic affinity and structural homology between Bergson and Debussy. Música em Contexto, 9(1), 55-88.

Hart, B. (2008). The symphony and national identity in early twentieth-century France. In B. Kelly (Ed.), French Music, Culture and Identity, 1870-1939 (pp. 131-148). Rochester, NY: University of Rochester Press.

Huron, D. (2001). What is a musical feature? Forte's analysis of Brahms's Opus 51, No. 1, revisited. Music Theory Online, 7(4).

Labov, W. (1972). Sociolinguistic patterns. Philadelphia, PA: University of Pennsylvania Press.

London, J., Polak, R., \& Jacoby, N. (2016). Rhythm histograms and musical meter: a corpus study of Malian percussion music. Psychon. Bull. Rev., 24, 474-480. https://doi.org/10.3758/s13423-016-1093-7

Meyer, L. (1989). Style and music: history, theory, and ideology. Philadelphia: PA University of Pennsylvania Press.

Pasler, J. (2007). A sociology of the apaches: 'sacred batallion' for Pelléas. In B. Kelly and K. Murphy (Eds.), Berlioz and Debussy: Sources, Contexts, Legacies (pp. 149-66). Burlington, VT: Ashgate.

Patel, A. (2008). Music, language, and the brain. Oxford, UK: Oxford University Press.

Robson, C. \& McCartan, K. (2015). Real world research (4th ed.). West Sussex, UK: Wiley.

Rogers, E. (2003). Diffusion of innovations ( $5^{\text {th }}$ ed.). New York: The Free Press.

Silverman, D. (1989). Art Nouveau in fin-de-siècle France: politics, psychology, and style. Berkeley, CA: University of California Press. https://doi.org/10.1525/9780520913288

Van der Weij, B., Pearce, M., \& Honing, H. (2017). A probabilistic model of meter perception: simulating enculturation. Frontiers in Psychology 8, 25-51. https://doi.org/10.3389/fpsyg.2017.00824

Van Leeuwen, T. (1999). Speech, music, sound. New York, NY: Palgrave McMillan. https://doi.org/10.1007/978-1-349-27700-1

Woldu, G. (2001). Debussy, Fauré, and d'Indy and conceptions of the artist: the institutions, the dialogues, the conflicts. In J. Fulcher (Ed.), Debussy and His World (pp. 235-253). Princeton: Princeton University Press. https://doi.org/10.1515/9781400831951-009 